\title{
Bridging Emergent Attributes and Darwinian Principles in Teaching Natural Selection
}

\author{
Dongchen $\mathrm{Xu}^{1, *}$, Michelene T.H. $\mathrm{Chi}^{2}$ \\ ${ }^{1}$ Department of Psychology, Arizona State University, USA \\ ${ }^{2}$ Mary Lou Fulton Teacher's College, Arizona State University, USA
}

Copyright $\mathrm{O} 2016$ by authors, all rights reserved. Authors agree that this article remains permanently open access under the terms of the Creative Commons Attribution License 4.0 International License

\begin{abstract}
Students often have misconceptions about natural selection as they misuse a direct causal schema to explain the process. Natural selection is in fact an emergent process where random interactions lead to changes in a population. The misconceptions stem from students' lack of emergent schema for natural selection. In order to help students construct a correct emergent schema: 5 inter-level attributes that explain the relationship between the micro-level interactions and macro-level patterns in emergent processes were used to develop learning materials for evolution. This new set of learning materials focusing on emergent attributes were compared to another set of learning materials focusing on Darwinian principles (the more traditional approach in teaching natural selection). In addition, a third set of learning material prompting relational thinking between Darwinian principles and emergent attributes were created. Results suggested that participants with higher prior knowledge of natural selection benefited more from this third relational approach, as they answered deep transfer questions more successfully than participants who received other materials that only focused on either emergent attributes or Darwinian principles.
\end{abstract}

Keywords Emergent Schema, Natural Selection, Prior Knowledge

\section{Introduction}

Science concepts involving emergent processes such as natural selection and diffusion are hard to understand correctly by people because they readily possess misconceptions on these processes [1,2]. Misconceptions in natural selection in particular are very robust and pervasive, to an extent that even college biology majors and pre-service science teachers retain such misconceptions $[3,4,5]$. The misconceptions in natural selection stem from using an inappropriate direct causal schema to interpret emergent processes $[6,7]$. For example, when thinking about the natural selection process of peppered moths getting darker after the Industrial Revolution, one misconception is thinking that the peppered moths transformed their genetic "essence" to adapt to a polluted darker environment [8]. This misconception as well as many other misconceptions in natural selection can be linked to thinking of a direct cause that singularly governs the change in the population (e.g. biological essence in this case). Misconceptions in emergent processes can therefore be attributed to adopting the wrong kind of direct causal schema and forming an inappropriate direct causal explanation. This kind of misconceptions are by its nature much harder to get rid of, because they are not caused by mistakes on certain details or facts; instead they are caused by using a fundamentally inappropriate mental model to explain some phenomena that are based on another type of model or system [1]. For that reason, misconceptions about natural selection have a more psychological basis rather than a biological basis, and constructing a correct emergent mental model is essential in reaching a correct representation of natural selection.

To understand an emergent process, one must understand the true nature of the interactions at the micro level, and how these interactions work together to cause the global pattern at the macro level. Chi and her colleagues [2] summarized five characteristics or attributes for inter-level causal relationships between micro-level interactions and macro-level pattern. 1) The global macro-level pattern arises from all the interactions at the micro-level, and not from just a subset of interactions or a specific interaction. 2) All interactions have equal status in their contribution to the pattern, in that no specific or subset of interactions is more special than others. 3) The interactions and the pattern can have disjoint behaviors, so sometimes interactions can go against the pattern. 4) Interactions are carried out to achieve local goals only disregarding the pattern. 5) The collective net effect of all these interactions causes the pattern. Since we will be referring to these 5 inter-level emergent attributes a lot, we will simply call them "emergent attributes" from now on.

The emergent attributes described above appear 
straightforward, but if we want to implement them to help students construct an emergent causal schema, simply showing these attributes would not be sufficient. For example, we can teach a student attribute \#3 on disjoint interactions, the student may remember what this attribute is and recall that interactions can be disjoint or go against the pattern. But how is this the case? What does this fact really do in the large picture, or what is the influence of having a few disjoint interactions in a process? All these are not clarified by simply showing the attribute. To address these concerns, we propose a method of teaching natural selection that combines the five emergent attributes with the more traditional way of teaching that involves "Darwinian principles." There are also five Darwinian principles and they can be briefly summarized as the following: 1) variation exists in a species; 2 ) only inheritable traits affect evolution; 3) some variations have a survival advantage; 4) survival advantage may lead to reproductive advantage; 5) small changes to the species due to differential reproduction accumulate over time and this is how evolution takes place [9].

We shall provide an example in which several emergent attributes are applied to generate and explain one Darwinian principle. With Darwinian principle \#1 on variation, there exists a teleological misconception which states that new traits appear in order to better adapt to an environment. If we apply this misconception to peppered moths, it would suggest that dark colored moths only appeared after the industrial revolution in order to match the darkened environment. In order to properly understand that a species such as peppered moths can already have variation of dark moths before the environment change, we could apply emergent attributes \#3 and \#4. Emergent attribute \#4 directly contradicts the teleological conception because it states that interactions serve local goals only and do not intend to cause the pattern. In this context, that means peppered moths would not "invent" a new trait in order to fit or better adapt to their environment. Emergent attribute \#3 on the other hand can explain why a dark variation of moths existed before environment got darker. The darker type of moths existed because interactions are random and can be disjoint the overall pattern, which was a predominantly light population in a cleaner environment before Industrial Revolution. Therefore, either through random mutation or mating with a darker individual, dark color moths were born even before the Industrial Revolution polluted the environment.

In the above example, Darwinian principles \#1 on variation is treated like a sub-process of natural selection that is also emergent. Although the principle of variation (principle 1) itself is not an emergent process, but how variation forms involves an emergent process. Another example is Darwinian principle \#3 on survival advantage, while the advantage itself is not an emergent process, how survival advantage emerges from interactions between species and environment can be considered an emergent process. These sub-processes can be treated as examples of emergence where emergent attributes can be applied to. By applying emergent attributes, people can understand how these Darwinian principles come to be. That way, their understanding of these principles is more accurate and they are less likely to form misconceptions. Emergent attribute become more relevant as well since a concrete example is provided in the form of Darwinian principles. In a sense, we could treat Darwinian principles as "examples" and emergent attributes as "rules". A combination of rules and examples would be most beneficial to learning because they can clarify each other [10].

Based on this assumption, we propose a study that compares the learning outcome of three conditions: a "biological condition" that focuses on teaching only the Darwinian principles; an "emergent condition" that focuses on teaching only the emergent attributes; and a "bridging condition" links both by prompting application of attributes to explain principles as well as reflection of the attributes.

The hypotheses are that subjects learning from bridging condition should demonstrate a deeper understanding of natural selection compared to the other two conditions; they should also be better at transferring what they have learned to different examples of natural selection.

\section{Materials and Methods}

\section{Participants}

Participants were all undergraduate students from a southwestern public university in the United States. They participated in the study to receive research credits for an introductory psychology class. There were 25 participants in the biological condition, 25 in the emergent condition and 37 in the bridging condition.

\section{Learning Materials and Assessments}

Introductory Text. The introductory text was very simple and provided necessary background information of peppered moths and Industrial Revolution for subjects. The main text described Darwinian principles and Emergent attributes in more detail. The text for Darwinian principles was mainly adapted from Ohlsson \& Bee [9] and the text for emergent attributes was mainly adapted from Chi, et al. [2]. The introductory text had 1714 words.

Pre- and Post-Tests. The pre-test contained 12 multiple choice questions with six questions focusing on Darwinian principles (referred to as "principle questions" from now on) and six questions focusing on emergent attributes (referred to as "attribute questions" from now on). The post-test included 22 questions in total. 12 of these questions were reused from the pre-test. Two new principle questions and two new attribute questions were added to the post-test. In addition, six questions from the Natural Selection Concept Inventory [11] were added to the post-test. The concept inventory questions were selected based on their difficulty. These questions served as deep questions as well as transfer questions because they involved different natural selection processes besides peppered moths. They will be referred to 
as "transfer questions" from now on.

Workbooks. Workbooks mostly consisted of open-ended thought questions that aimed at scaffolding certain concepts. The scaffolding usually involved prompting participants to make a claim in a given context, and then prompting them to back up the claim via explanation. These thought questions were organized by several sets of questions that each had a different focused concept. For the biological condition, each set of questions focused on one Darwinian principle and there were five sets of questions. Similarly, the workbook for emergent condition had five sets of questions that each focused on one emergent attribute. For the bridging condition, each set focused on using several emergent attributes to generate or describe one Darwinian principle. There were only four sets of questions of bridging condition because Darwinian principle \# 2 on inheritable traits does not involve any emergent process. The biological workbook had 1373 words; the emergent workbook had 1431 words; the bridging workbook had 1460 words.

\section{Procedure}

Participants started with the introductory text and the pre-test. After they finished reading the introductory text and completed the pre-test, those materials were collected and they were given the main reading text. After they read the main text, they were each given one version of the workbook based on their conditions. They were allowed to use the main reading text as a reference in this process. After they finish the workbook, the workbook and the main reading text were collected and participants were given the post-test. Finishing the post-test ends the participation of this experiment. The full length of the experimental session was 90 minutes.

\section{Mean Split of Participants Based on Pre-test}

Due to time constraint, no feedback was given to participants as they worked through the workbooks. For that reason, learning ability and prior knowledge may confound how much participants learn from the treatment. A split based on mean pre-test score of all participants was carried and it separates participants into "high prior knowledge participants" and "low prior knowledge participants". ANOVA analyses were carried out for both groups on their pre-test and post-test scores.

After the mean split by pre-test, for high prior knowledge participants, there were 11 participants in biological condition, 11 participants in emergent condition and 16 participants in bridging condition; for low prior knowledge participants, there were 13 participants in biological condition, 12 participants in emergent condition and 20 participants in bridging condition.

\section{Results}

ANOVA that compared overall pre-test score between high prior knowledge participants $(\mathrm{n}=38)$ and low prior knowledge participants $(\mathrm{n}=45)$ indicated that a significant difference existed between high and low groups (high $>$ low), $F(1,81)=186.88, p<.01$, but not within each group across conditions. ANOVA that compared just principle question scores or just attribute question scores showed similar results between the 2 groups, $F(1,81)=98.46, p<.01$ (principle question scores), $F(1,81)=81.22, p<.01$ (attribute question scores).

Within the high prior knowledge participants, ANOVA on post-test scores indicated 2 significant differences across conditions. For principle question scores, there was also a significant difference across conditions, $F(2,35)=3.54, p$ $=.04$. Post-hoc Tukey's HSD showed that biological condition had marginally higher principle questions score compared to emergent condition $(p=.06)$, bridging condition also had marginally higher principle questions score compared to emergent condition $(p=.07)$. With transfer questions, there was also a significant difference across conditions, $F(2,35)=4.35, p=.02$. Post-hoc Tukey's HSD showed that bridging condition had significantly higher transfer question scores compared to biological condition ( $p$ $=.02)$. (See Figure 1) 


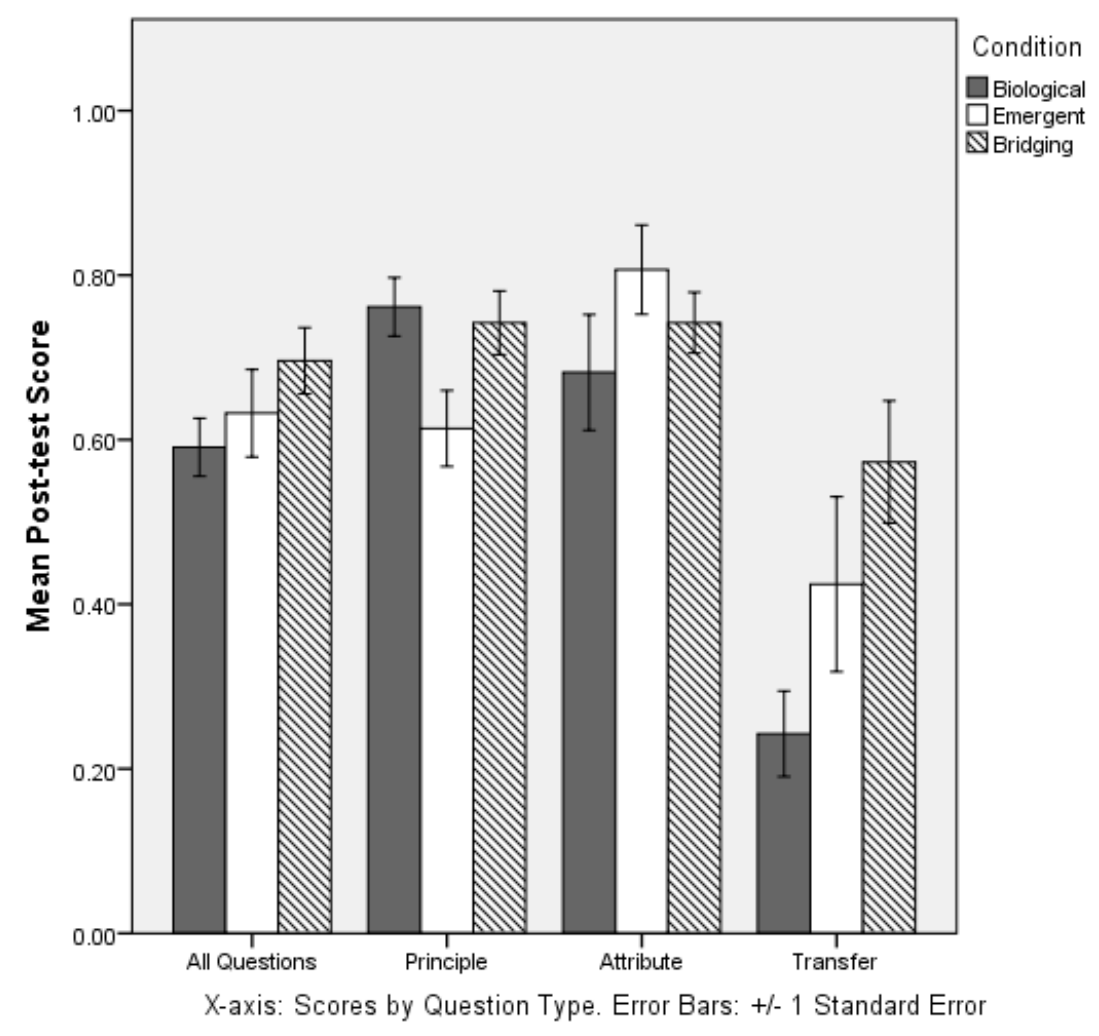

Figure 1. Post-test Results for High Prior Knowledge Participants

Within the low prior knowledge participants, ANOVA on post-test scores only found a significant difference on principle question scores across conditions, $F(2,42)=8.22, p<.01$. Post-hoc Tukey's HSD showed that biological condition had higher score on principle questions than both emergent condition $(p<.01)$ and bridging condition $(p=.03)$. (See Figure 2$)$

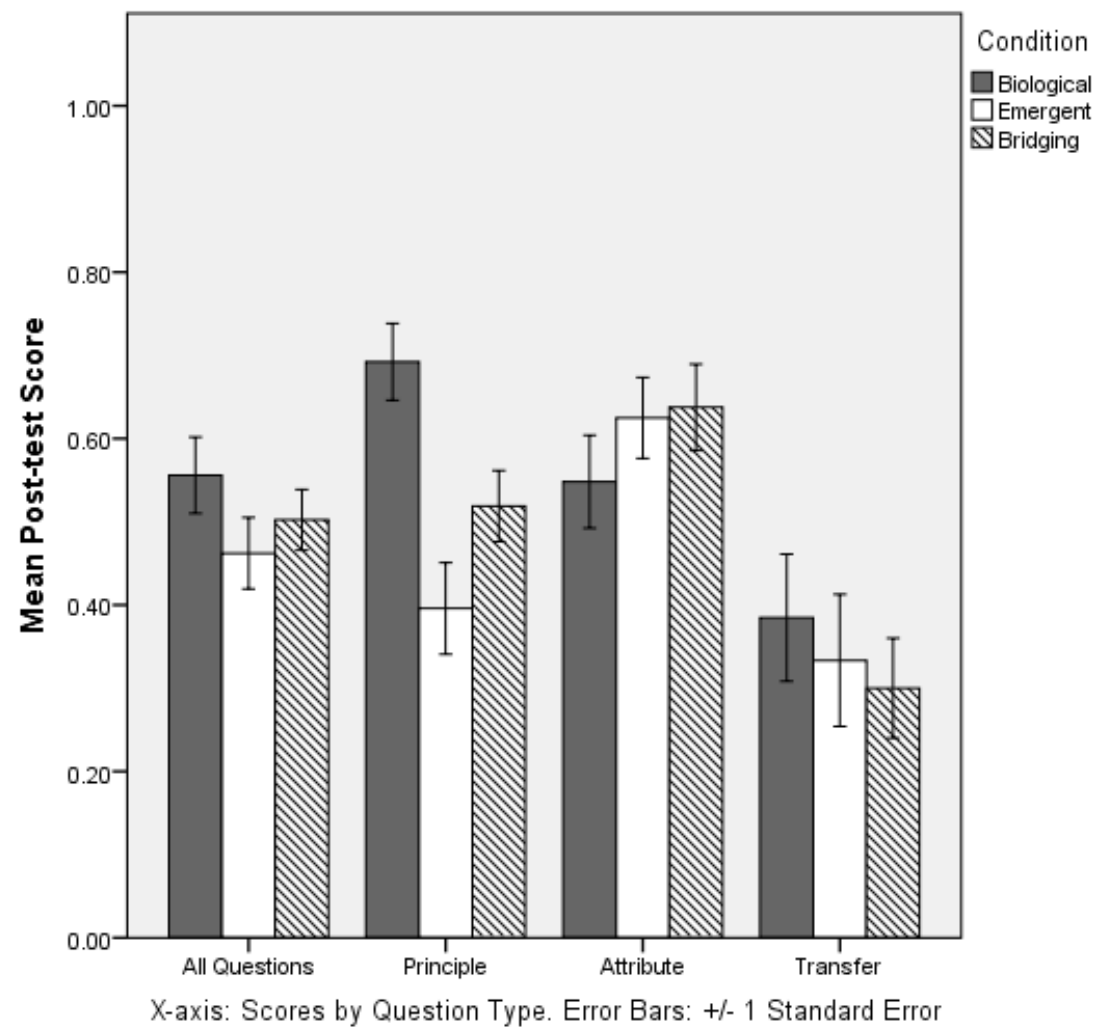

Figure 2. Post-test Results for Low Prior Knowledge Participants 


\section{Discussion}

High and low prior knowledge participants benefited in different ways from different conditions based on the results. For the high prior knowledge group, they benefited more from the bridging condition as they learned more deep knowledge and were able to transfer them better. For the low prior knowledge group, they benefited more from the biological condition as they learned Darwinian principles better. This finding may be explained by Schwartz's and Bransford's finding [12] where students who analyzed contrasting examples learned more from later lectures. The explanation is that people must be prepared and have a knowledge structure ready before they can understand sophisticated explanations of certain concepts. In Schwartz's and Bransford's study [12], analyzing contrasting cases provided that knowledge structure.

In this study, the biological condition focused on Darwinian principles, which essentially were "descriptions" of different processes within natural selection; the emergent condition focused on emergent attributes, which essentially were "rules" that govern the mechanism of natural selection; the bridging condition however, via focusing on how to apply emergent attributes to Darwinian principles, essentially provided "explanations" to the difficult aspects of understanding natural selection. Therefore, based on Schwartz's and Bransford's results [12], people need to have some differentiated knowledge structure before they can appreciate the "explanations" that can be found in the bridging condition. This could potentially be why high prior knowledge participants benefited more from bridging condition than low prior knowledge participants. On the other hand, when participants had low knowledge of natural selection in the beginning, the concrete "descriptions" of the process provided by Darwinian principles would make more sense than the "rules" or "explanations".

This study has two potential contributions to the teaching of natural selection. First contribution is on how to incorporate emergent concepts in the teaching of natural selection: by application of emergent attributes to different Darwinian principles. Second contribution is on when to incorporate emergent concepts in teaching natural selection: that emergent concepts are better introduced after some Darwinian concepts are taught. Furthermore, this study may also shed light on how to teach other scientific processes that involves emergence such as diffusion and heat transfer. From a practice perspective, the workbooks involved in this study only took students on average 29 minutes to finish $(S D=11)$, so it is something that can be implemented within a class period that can improve students' understanding of natural selection. Overall this study provides some much needed implications in improving the teaching of natural selection, a concept that is poorly understood even today.

\section{REFERENCES}

[1] Chi MT. Commonsense conceptions of emergent processes: Why some misconceptions are robust. The journal of the learning sciences. 2005 Apr 1;14(2):161-99.

[2] Chi MT, Roscoe RD, Slotta JD, Roy M, Chase CC. Misconceived causal explanations for emergent processes. Cognitive science. 2012 Jan 1;36(1):1-61.

[3] Gregory TR. Understanding natural selection: essential concepts and common misconceptions. Evolution: Education and Outreach. 2009 Jun 1;2(2):156-75.

[4] Moore R, Mitchell G, Bally R, Inglis M, Day J, Jacobs D. Undergraduates' understanding of evolution: ascriptions of agency as a problem for student learning. Journal of Biological Education. 2002 Mar 1;36(2):65-71.

[5] Nehm RH, Reilly L. Biology majors' knowledge and misconceptions of natural selection. BioScience. 2007 Mar 1;57(3):263-72.

[6] Penner DE. Explaining systems: Investigating middle school students' understanding of emergent phenomena. Journal of Research in Science Teaching. 2000 Oct 1;37(8):784-806.

[7] Ferrari M, Chi MT. The nature of naive explanations of natural selection. International Journal of Science Education. 1998 Dec 1;20(10):1231-56.

[8] Shtulman A. Qualitative differences between naïve and scientific theories of evolution. Cognitive psychology. 2006 Mar 31;52(2):170-94.

[9] Ohlsson S, Bee NV. The effect of expository text on children's explanations of biological evolution. Office of Educational Research and Improvement (OERI) report. Learning Research and Development Center, Pittsburgh. 1992.

[10] Goldstone RL, Wilensky U. Promoting transfer by grounding complex systems principles. The Journal of the Learning Sciences. 2008 Oct 22;17(4):465-516.

[11] Anderson DL, Fisher KM, Norman GJ. Development and evaluation of the conceptual inventory of natural selection. Journal of research in science teaching. 2002 Dec $1 ; 39(10): 952-78$.

[12] Schwartz DL, Bransford JD. A time for telling. Cognition and instruction. 1998 Dec 1;16(4):475-5223. 